\title{
3D Printing of NinjaFlex Filament onto PEDOT:PSS-Coated Textile Fabrics for Electroluminescence Applications
}

\author{
MELKIE GETNET TADESSE (1), $, 2,3,4,5,6$ DELIA DUMITRESCU, ${ }^{1}$ \\ CARMEN LOGHIN, ${ }^{2}$ YAN CHEN,${ }^{3}$ LICHUAN WANG,${ }^{3}$ \\ and VINCENT NIERSTRASZ ${ }^{1}$ \\ 1.-Textile Materials Technology, Department of Textile Technology, Faculty of Textiles, Engi- \\ neering and Business, University of Borås, 50190 Borås, Sweden. 2.-Faculty of Textiles, Leather \\ and Industrial Management, Gheorghe Asachi Technical University of Iasi, 53, D. Mangeron Blv., \\ 700050 Iasi, Romania. 3.-The College of Textile and Clothing Engineering, Soochow University, \\ 178 G.J. D. Road, Suzhou 215021, China. 4.-e-mail: melkie.tadesse@hb.se. 5.—e-mail: \\ melkiegetnet23@gmail.com.6.-e-mail: tadesse.melkie@tuiasi.ro
}

Electroluminescence (EL) is the property of a semiconductor material pertaining to emitting light in response to an electrical current or a strong electric field. The purpose of this paper is to develop a flexible and lightweight EL device. Thermogravimetric analysis (TGA) was conducted to observe the thermal degradation behavior of NinjaFlex. Poly(3,4-ethylenedioxythiophene):poly(styrene sulfonic acid)_PEDOT:PSS—with ethylene glycol (EG) was coated onto polyester fabric where NinjaFlex was placed onto the coated fabric using three-dimensional (3D) printing and phosphor paste, and BendLay filaments were subsequently coated via 3D printing. Adhesion strength and flexibility of the 3D-printed NinjaFlex on textile fabrics were investigated. The TGA results of the NinjaFlex depict no weight loss up to $150^{\circ} \mathrm{C}$ and that the NinjaFlex was highly conductive with a surface resistance value of $8.5 \mathrm{ohms} / \mathrm{sq}$.; the coated fabric exhibited a uniform surface appearance as measured and observed by using four-probe measurements and scanning electron microscopy, respectively, at 60\% PEDOT:PSS. The results of the adhesion test showed that peel strengths of $4160 \mathrm{~N} / \mathrm{m}$ and $3840 \mathrm{~N} / \mathrm{m}$ were recorded for polyester and cotton specimens, respectively. No weight loss was recorded following three washing cycles of NinjaFlex. The bending lengths were increased by only a factor of 0.082 and 0.577 for polyester and cotton samples at 0.1-mm thickness, respectively; this remains sufficiently flexible to be integrated into textiles. The prototype device emitted light with a $12-\mathrm{V}$ alternating current power supply.

Key words: NinjaFlex, adhesion test, 3D printing, electroluminescence, TGA, emitter

\section{INTRODUCTION}

A new generation of textile, specifically wearable electronics, demands materials that can be directly worn on the curved human body. Recently, textilebased wearable electronics (e-textiles) have

(Received June 5, 2017; accepted December 7, 2017; published online December 19, 2017) exhibited excellent promise in this regard and have been produced by coating with intrinsically conductive polymers (ICPs) for several applications such as polyaniline, ${ }^{1}$ polypyrrole, ${ }^{2}$ and PEDOT:PSS. ${ }^{3}$ Therefore, e-textiles have received great attention. Among these, PEDOT:PSS has been used for various applications due to its electromechanical properties. $^{4}$ Zeng et al. ${ }^{5}$ claimed the use of PEDOT:PSS using fiber-based wearable electronics as e-textiles. They discussed the issue of being flexible, 
lightweight, and comfortable since these properties are very important for wearable applications. The PEDOT:PSS conductivity trend and its effect on the stretchable and flexible electrochromic property has been investigated using various textile fabrics as revealed by Ding et al. ${ }^{6}$; the electronic properties of the stretchable substrate ${ }^{7}$ has been also explored. Stretchable heaters have been produced using PEDOT:PSS polymer as a conductive material using textile fabric substrate. ${ }^{8}$ Cost-effective stretchable heaters have been produced using sodium dodecyl sulfate as a conductivity enhancer.

Among these e-textiles, electroluminescence (EL), an optical and electrical property in which a part emits light in response to the passage of electrical current, has been recently paid attention since the introduction of an organic EL device in 1987 by CW Tang. ${ }^{9}$ Later in 1995 , Chihaya et al. reported the molecular design of hole-injection transport materials for durable organic EL diodes ${ }^{10}$ and for flexible lamps. ${ }^{11}$ EL can be realized by novel phosphorescent orange-light-emitting complex (bzq) $)_{2} \operatorname{Ir}($ dipba) and a blue host emitter ${ }^{12}$ as well as undoped organic light-emitting devices using aluminum tris (8quinolinolato) chelates. ${ }^{13}$ Highly efficient and moisture insensitive photoluminescence material has been produced ${ }^{14,15}$ using perovskite materials. However, these materials are not flexible, stretchable, and are mechanically fragile. In addition, organic polymers such as polythiophene, cyanopolymers, polyphenylenes, silicon-containing polymers, polyfluorenes, and polymers with triple bonds in the main chain have been used to synthesize EL materials. ${ }^{16}$

The main components of EL devices are the rear electrode, dielectric material, light-emitter, and front electrode. ${ }^{17,18}$ PEDOT:PSS has been used to produce EL devices ${ }^{17,19,20}$ in addition to other applications such as self-healing, ${ }^{21}$ solar cells, ${ }^{22,23}$ and polymer photovoltaic cells. ${ }^{24}$ On the other hand, in the EL device fabrication systems, PEDOT:PSS has been used to enhance hole injection using tin(II) fluoride $\left(\mathrm{SnF}_{2}\right)^{25}$ in addition to serving as an anode and transparent electrode. ${ }^{19}$ Therefore, these components should be carefully selected and designed in order to convert the non-thermal energy into light energy.

EL application requires a flexible nature so that it could easily integrate with wearable textile materials. $^{26}$ Mechanisms of light emission have been investigated using various electroluminescent polymers to produce more flexible materials. ${ }^{16}$ Among them: conjugated ${ }^{27}$ and self-assembled ${ }^{28}$ polymers have been used in EL applications. Polymer lightemitting devices of considerably small size and varied patterns have been adopted and used in the construction of flexible displays. ${ }^{29}$ EL structures have been screen-printed onto elastic substrates to replace polymer foils and indium tin oxide (ITO) using poly(methyl methylacrylate $)^{30}$; however, damages were found upon bending. Flexibility and weightless properties are the key considerations in coupling with textile materials. In spite of this fact, using a copolymer, EL and photoluminescence applications have been developed. ${ }^{31}$

When electrical conductivity is applied to textile materials, it is possible to create intelligent materials with a wide range of applications. Electrical conductivity can be applied onto textile materials through various methods such as screen printing, ${ }^{32}$ coating, ${ }^{33}$ and inkjet printing. ${ }^{34}$ Among these, the coating process has been widely used not only for its easy application but also because coated textiles can be used in various applications such as sensors, data transfer, data monitoring, corrosion protection, and electromagnetic interface (EMI) shielding. ${ }^{33}$ Moreover, textile coating has been used for producing flexible, lightweight, and stretchable functional textiles. ${ }^{35}$

Most recently, three-dimensional (3D) printing technique has been used for the production of smart materials using a textile material as the substrate. There are many materials that have been used for 3D printing, of which, acrylonitrile-butadienestyrene (ABS), polylactic acid (PLA), nylon, and NinjaFlex are most prominent. ${ }^{36}$ NinjaFlex, which is the focus of this paper, has been used in the production of antennas ${ }^{37-39}$ and in microwave applications. ${ }^{40}$ In this study, NinjaFlex was selected to fabricate the EL device due to its high flexibility and good dielectric properties, as discussed in Ref. 41. Flexible and stretchable polymers such as NinjaFlex have been investigated for use in soft electronics applications using 3D printing. ${ }^{42}$

EL devices are manufactured in various sequences depending on the types of materials and methods used. It has been claimed that a single-layer EL display can be fabricated using PEDOT:PSS and ITO films as the anode (rear electrode), aluminum as the front electrode, and using a chloroform solution of poly[2-methoxy-5-(2-ethylhexyloxy)-1,4-phenylenevinylene]-MEH-PPV - as the EL material using spin coating. ${ }^{19}$ Similarly, a device has been fabricated using silver paste as the rear electrode, PEDOT:PSS as the transparent electrode, barium titanate as the dielectric material, and zinc sulfide as the emitter. ${ }^{17}$ However, the dielectric material, barium titanate, is a ceramic material that is easily fractured before plastic deformation. Moreover, inorganic EL displays have been fabricated using ITO as the bottomemitting display, polyester ethylene terephthalate (PET) substrates (DuPont Teijin) as the top-emitting display, and phosphor paste as the luminescent material. ${ }^{43}$ In contrast, organic EL devices have been fabricated using $\mathrm{ITO}^{44,45}$ and a gold (Au) electrode ${ }^{46}$ on a glass substrate.

However, fabrication of dielectric materials using inorganic or organic materials is considerably expensive, in addition to their front electrodes (primarily low-work function alloys) being susceptible to corrosion. Additionally, the anode component of most organic electroluminescent devices, ITO, yields a relatively inert contact and is costly. ${ }^{47} \mathrm{In}$ order to reduce the cost of materials and to create 
sustainable systems, stretchable and flexible polymeric materials, particularly low-cost, non-brittle, and lightweight materials, should be investigated.

In this paper, we demonstrated 3D printing and a coating procedure to be applied onto flexible substrates to produce a single-unit EL device. This enabled us to use a 3D printer to fabricate flexible dielectric material on conductive textiles produced by coating. To demonstrate the EL device, NinjaFlex was 3D printed onto a PEDOT:PSS-coated polyester fabric to serve as the non-brittle dielectric material. The PEDOT:PSS-coated substrate was thus used as the rear electrode (anode). Phosphor blue paste, used as an emitter, was coated onto 3Dprinted NinjaFlex; lastly, the knitted copper fabric was used as the front electrode (cathode), which was covered by a non-conductive and transparent polymer, BendLay filament, via 3D printing. A sample prototype of the proposed EL device was thus fabricated for demonstration purposes.

\section{EXPERIMENTAL}

\section{Materials}

NinjaFlex, a thermoplastic polyurethane (TPU), purchased from Creative Tools (with $\emptyset: 1.75 \mathrm{~mm}$; specific gravity: $1.19 \mathrm{~g} / \mathrm{cc}$; elongation at break: $660 \%$; low-tack, easy-to-feed texture) was used as the 3D printing material using a WANHAO 3D printer machine. ZEHNTNER ZUA 2000, a universal paint applicator, with adjustable gap height was used for the coating application. Plain weave polyester fabric (with gsm: 159; average thickness: $0.3 \mathrm{~mm}$; ends/cm: 30; and picks/cm: 22) and cotton fabric (with gsm: 130; average thickness: $0.29 \mathrm{~mm}$; ends/cm: 30; picks/cm: 26 ), heat-set and bleached by the supplier were used in adhesion and bending testing via universal tensile testing and Shirley stiffness testing machines, respectively. Phosphor blue paste purchased from Gwent Group (viscosity: $0.86-2.50 \mathrm{~Pa} \mathrm{~s}$; at $25^{\circ} \mathrm{C}$; solid content: $80-82.5 \%$ at $150^{\circ} \mathrm{C}$ ) was used as an emitter material. A PEDOT:PSS aqueous solution (Heraeus $\mathrm{GmbH}$, Germany) with a concentration of $1.3 \%$ by weight and PSS-to-PEDOT ratio of $2: 5$ was used as the conducting polymer coated onto the polyester fabric serving as the rear electrode. Plain knitted copper fabric with a diameter of $0.1 \mathrm{~mm}$ and an electrical resistivity of $2.5 \Omega / \mathrm{m}$ was used as the front electrode. Gel L75 N (Borchers GmbH, Germany), a hydrophobically modified ethoxylated urethane with $48 \%$ solid content, was used as the rheology modifier. Ethylene glycol (EG; Sigma Aldrich) with a $99.8 \%$ concentration was used as the conductivity enhancer.

\section{METHODS}

\section{Rear electrode preparation}

The rear electrode was produced by coating. EG (5\%), Gel L75N (6\%), and various concentrations of PEDOT:PSS were mixed using a mechanical stirrer at a speed of $600 \mathrm{rpm}$ for $10 \mathrm{~min}$. The variation in the concentration of PEDOT:PSS to the total solution was compensated for by varying the concentration of the rheology modifier. The coating of the polyester fabric with PEDOT:PSS was performed via a coating applicator with $200-\mu \mathrm{m}$ gap height with sample sizes of $10 \mathrm{~cm} \times 10 \mathrm{~cm}$ produced. Samples were dried at $100^{\circ} \mathrm{C}$ for $30 \mathrm{~min}$ and subsequently cured at $150^{\circ} \mathrm{C}$ for $5 \mathrm{~min}$. The four-probe surface resistance measurement principle was used to measure the electrical properties. Due to the deformability and irregularity of the textile surface structures, a $2-\mathrm{kg}$ weight was laid on the top of the probe to improve the contact between the textile material and the measuring device. In addition, samples were carefully placed on a soft insulating body in which a teethed-edge brass plate of the surface probe was placed to measure the surface resistance.

\section{Scanning electron microscopy (SEM) images}

SEM images were obtained with a Leo Ultra 55 scanning electron microscope equipped with a field emission gun (LEO Electron Microscopy Group, Germany) and a secondary electron detector. The acceleration voltage was $3 \mathrm{kV}$. Samples were cut with a razor blade. All samples were sputtered with gold.

\section{Thermal measurements}

Thermal behavior analysis of the NinjaFlex polymer was carried out using thermogravimetric analysis (TGA) to observe the thermal stability. A thermobalance TGA (Q500-1763) ramp at 20$600^{\circ} \mathrm{C}$ at a rate of $20^{\circ} \mathrm{C} / \mathrm{min}$ heated under nitrogen was used to investigate the mass loss/degradation temperature of the NinjaFlex.

\section{D printing and phosphor paste coating}

NinjaFlex, a flexible thermoplastic polyurethane, was printed via 3D printing onto PEDOT:PSScoated polyester fabric using a $3 \mathrm{D}$ printer machine. $3 \mathrm{D}$ scaffolds were designed using $\mathrm{CAD}$ software (rhinoceros 5), then designs were exported using a stereolithographic format (stl.file format) and uploaded to simplify the 3D software output, which was modified to print parameters such as infill percentage, printing speed, temperature, and sample size. Lastly, $5 \mathrm{~cm} \times 5 \mathrm{~cm} \times 0.1-\mathrm{mm}$ sample sizes were printed using the $3 \mathrm{D}$ printing technique. Printing was executed at a $230^{\circ} \mathrm{C}$ extrusion temperature and a $30^{\circ} \mathrm{C}$ build plate temperature, at a printing speed of $800 \mathrm{~mm} / \mathrm{min}$ with $100 \%$ infill percentage and $45^{\circ}$ orientation angle. After 3D printing of NinjaFlex, phosphor blue paste was coated on the top of the 3D-printed NinjaFlex at a $50-\mu \mathrm{m}$ gap height using a paint applicator. The phosphor blue paste was mechanically stirred at $600 \mathrm{rpm}$ for $5 \mathrm{~h}$ before coating to ensure 
homogenous mixing. After coating, curing was performed at $130^{\circ} \mathrm{C}$ for $15 \mathrm{~min}$. BendLay filament was $3 \mathrm{D}$-printed on top of the phosphor paste in a similar way where the copper fabric was sandwiched between phosphor blue paste and BendLay filament.

\section{Bending length measurement}

The bending lengths of the 3D-printed NinjaFlex on cotton and polyester fabrics and the EL device of varied thicknesses were measured according to the standards of ASTM D1388-2007 and BSI BS 33561991 (AMD 6337).

\section{Adhesion tests}

The 3D-printed NinjaFlex on cotton and polyester fabrics with $0.3-\mathrm{mm}$ thickness were prepared to measure the adhesion strength. Samples were produced for the T-peel testing for flexible-to-flexible bonded assemblies according to SS-EN ISO 11339:2010. The tests were performed using a tensile testing machine according to the ASTM D5034 fabric traction (grab) general testing method using a load cell of $5 \mathrm{kN}$. In addition to the interfacial adhesion, the durability of the surface adhesion of NinjaFlex against washing was checked using domestic laundry washing and drying procedures of type $3 \mathrm{~A}$ reference washing for textile testing (ISO 6330:2012); these procedures were performed to observe the durability of 3D-printed NinjaFlex with $100 \%$ polyester ballast against washing.

\section{Luminescent device fabrication and test}

There are several device fabrication methods; among these, the rear electrode-dielectric-emitterfront electrode arrangement was chosen, which suits our material systems. First, the NinjaFlex flexible filament was printed via the 3D printing machine on top of the PEDOT:PSS-coated polyester fabric. Then phosphor blue paste was coated on top of the NinjaFlex. The conductive copper fabric was subsequently fabricated via weft knitting. Finally, to prevent shorting and to ensure sufficient contact with the phosphor paste, the BendLay filament was 3D-printed on top of the copper fabric. Alternating current (AC) was applied using a power supply (-S350-12) of $12 \mathrm{~V}$ and 29-ampere $\mathrm{AC}$ input at 50$60 \mathrm{~Hz}$ to observe the luminescent light.

\section{RESULTS AND DISCUSSION}

The EL device was developed using NinjaFlex and PEDOT:PSS by integrating 3D printing and coating technologies. This EL device yields the advantage of being flexible, cost-effective, and lightweight. Thus, it could be easily integrated into wearable e-textiles. The 3D-printed NinjaFlex exhibited excellent durability against adhesion and has good flexibility.

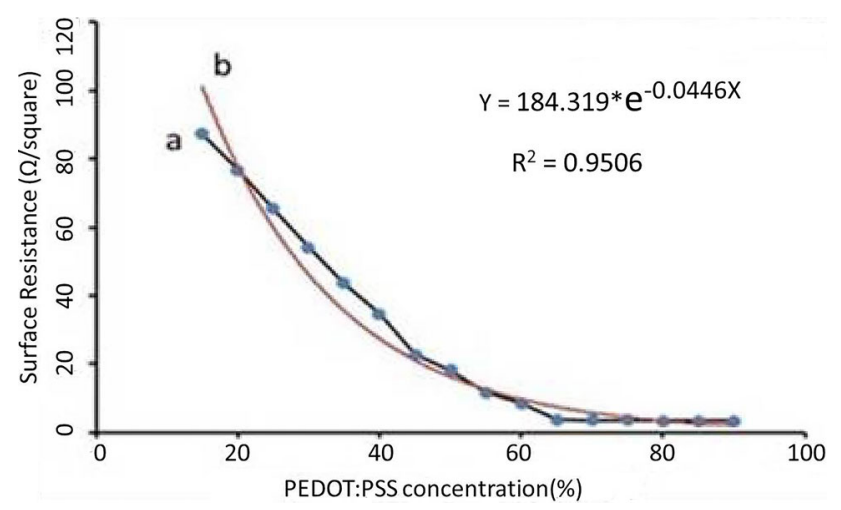

Fig. 1. Surface resistance: (a) actual value and (b) predicted value.

Thermal analysis of the filament yields promises for application as the dielectric material.

Intrinsically conductive PEDOT:PSS aqueous dispersion coated onto a polyester fabric was produced by mixing with EG to enhance the conductivity. The conductivity enhancement and durability against washing PEDOT:PSS-coated polyester fabric using EG has been reported in our work. ${ }^{48}$ In spite of the sensitivity of polymer-based coatings to humidity, samples were reasonably conductive after ten successive washing cycles, as discussed in our previous work. The washing durability of conductively coated fabrics allows integrating this kind of conductive material with wearable electronics for smart textile applications. The surface resistance with various concentration of PEDOT:PSS (weight percentage of PEDOT:PSS to the total prepared solution) is demonstrated in Fig. 1. The result was predicted by a non-linear regression model using Excel 2010 and it shows an excellent fitting curve against the fitting model. As shown in Fig. 1, 60\% PEDOT:PSS was observed to be a critical concentration point beyond which an insignificant change of the surface resistance occurs by increasing the PEDOT:PSS concentrations. The coatings can be regarded as electrically conductive with a surface resistance value of $87.3 \Omega / \mathrm{sq}$. at 15 wt.\% of PEDOT:PSS. The percent weight increases of the PEDOT:PSS-coated fabric for both polyester and cotton fabric were from $4 \%$ to $5 \%$ and the surface resistance values of samples produced using polyester and cotton fabrics were close. However, polyester fabric was selected for electroluminescent application due to its physical properties, and because acidic PEDOT:PSS will degrade the cellulose more than polyester fabric, as discussed in Ref. 49. In addition to its physical properties, polyester fabric is more hydrophobic than cotton. Therefore, polyester fabric is more resistant to moisture degradation than cotton fabric. Light output deteriorates with time and it becomes faster when the EL material is subjected to the environment. This is why polyester fabric was selected for the EL application. 

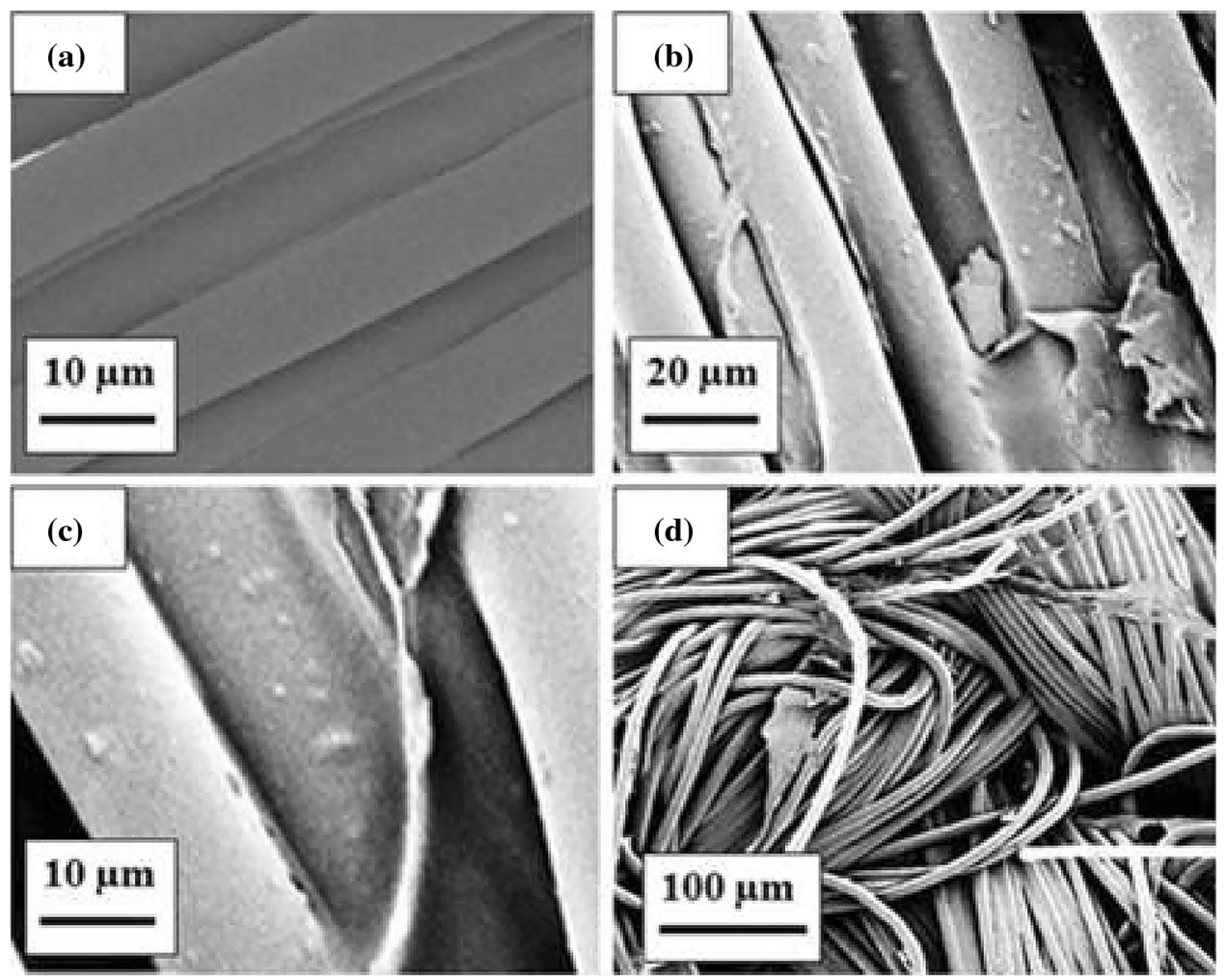

Fig. 2. Longitudinal SEM images of (a) uncoated and (b-d) coated samples.

The surface resistances of the coated polyester fabric were measured by applying the principles of four-probe measurement; it was subsequently recorded as $8.5 \mathrm{ohms}$ per square at 60\% PEDOT:PSS by taking three measurements; the average was reported. Electrical characterization during the fabrication of EL devices is essential for the sample to be used as a rear electrode. The conductivity of the coated fabric in this study is found to be adequate for use as a rear electrode.

In order to confirm the surface morphology changes of the coated samples, SEM images were taken in longitudinal and cross-sectional view. The longitudinal and cross-sectional views obtained by the SEM images of the samples before and after coating applications are demonstrated in Figs. 2 and 3, respectively. The spherical morphology of the PEDOT:PSS particles in longitudinal and crosssectional views, as shown in Figs. $2 b, c, d$ and $3 c, d$, respectively, show that the PEDOT:PSS particles were dispersed uniformly, homogeneously, and with relatively rough surfaces without critical agglomeration, whereas the uncoated sample possessed a relatively smooth surface morphology as there was no coating material deposited. The uncoated sample is represented by Figs. $2 \mathrm{a}$ and $3 \mathrm{a}, \mathrm{b}$. The circle-like morphology appears to be a distinctive and common structural characteristic of polyester fabric. The SEM images clearly distinguish the uncoated from coated samples.
The SEM images also show PEDOT:PSS is highly integrated into the structure of the fabric which helps retain stability of the PEDOT:PSS-coated sample when subjected to the environment. There are some small agglomerates detected on the surface. The reasonable explanation could be the rheology modifier solution creates these small dots due to mixing and properties of the rheology modifier.

TGA was used to measure mass loss versus temperature in a previous study. ${ }^{50}$ Materials for the electroluminescent devices are required to have thermal and environmental stability. To evaluate the thermal stability of NinjaFlex, TGA measurements were conducted. Figure 4 demonstrates the thermal decomposition properties of the NinjaFlex polymer under nitrogen. Only $0.84 \%$ weight loss occurs at approximately $294^{\circ} \mathrm{C}$. Maximum weight losses of the polymer occurred approximately between $270^{\circ} \mathrm{C}$ and $390^{\circ} \mathrm{C}$. A $93 \%$ weight loss of the sample is observed by approximately $450^{\circ} \mathrm{C}$. The results of the experiment showed that NinjaFlex was thermally stable up to $150^{\circ} \mathrm{C}$ without any weight loss, exhibiting no indications of melting and weight loss. Starting from $150^{\circ} \mathrm{C}$, a very small fraction of weight loss was observed. Simply put, NinjaFlex was unaffected by the environment (nitrogen or oxygen) up to $150^{\circ} \mathrm{C}$, as there were no indications of physical transition as demonstrated by weight loss up to this temperature. 

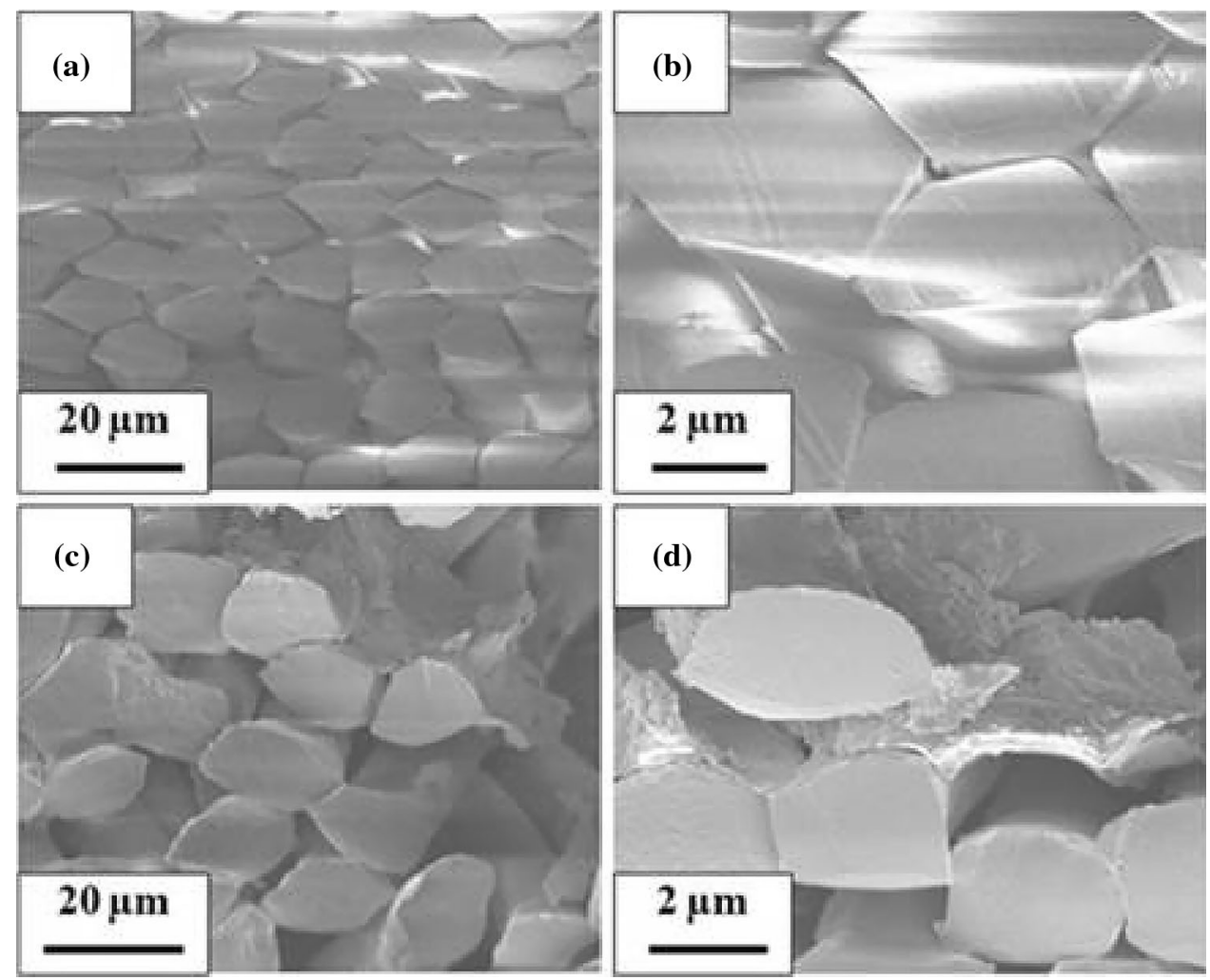

Fig. 3. Cross-sectional SEM images of ( $a$ and b) uncoated and (c and d) coated samples.

Using 3D printing equipment, samples were produced at extruder and platform temperatures of $230^{\circ} \mathrm{C}$ and $30^{\circ} \mathrm{C}$, respectively. The infill percentages were set to $40,50,70,80$, and 100 , with $100 \%$ infill being selected for the subsequent application. This selection was made because $100 \%$ infill inhibited the penetration of phosphor paste into the PEDOT:PSS-coated fabric. Since the filament is considerably flexible, clogging was a problem, as observed during printing. Subsequently, in order to prevent this problem, the printing speed was varied by using $400 \mathrm{~mm} / \mathrm{min}, 500 \mathrm{~mm} / \mathrm{min}, 800 \mathrm{~mm} / \mathrm{min}$, $1000 \mathrm{~mm} / \mathrm{min}$, and $1200 \mathrm{~mm} / \mathrm{min}$. The clogging problem was optimized at $800 \mathrm{~mm} / \mathrm{min}$ and this speed was used throughout this paper. It is wellknown that as the printing thickness of the NinjaFlex increases, the penetration of phosphor paste will be reduced. Considering this, $0.1-\mathrm{mm}, 0.2-\mathrm{mm}$, and $0.3-\mathrm{mm}$ printing thicknesses were tested and the $0.3-\mathrm{mm}$ thickness of the $3 \mathrm{D}$ printing was selected due to adhesion strength. BendLay filament was printed onto copper fabric by the same printing procedure. Lastly, phosphor paste was prepared and cured according to the supplier information and subsequently coated onto the 3Dprinted NinjaFlex using a paint applicator with a gap height of $50 \mu \mathrm{m}$. The 3D-printed NinjaFlex was used to prevent the catastrophic dielectric breakdown between the phosphor layer and electrodes.

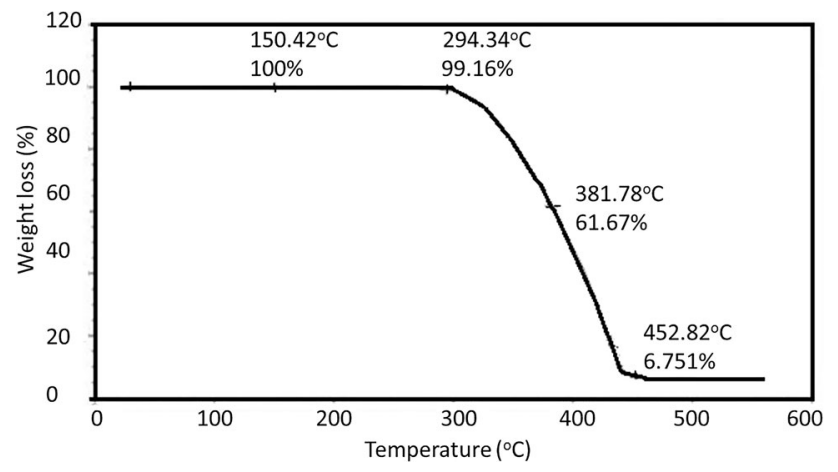

Fig. 4. Thermogravimetric analysis of NinjaFlex.

The restrictions in flexing behavior of the textile materials have been respectively characterized by measuring the bending lengths of each of the materials. ${ }^{51}$ The bending lengths of the 3D-printed NinjaFlex on polyester and cotton fabrics with printing thicknesses of $0.1 \mathrm{~mm}$ and $0.3 \mathrm{~mm}$ were measured using a Shirley stiffness tester and recorded as illustrated in Fig. 5. Additionally, the bending lengths of the EL devices with thicknesses of $1.1 \mathrm{~mm}$ and $1.3 \mathrm{~mm}$ were measured. A total of six measurements were taken on the 3D-printed sample and on the rear surface of the sample; the average was reported. Comparisons were made between the control fabric and the 3D-printed 


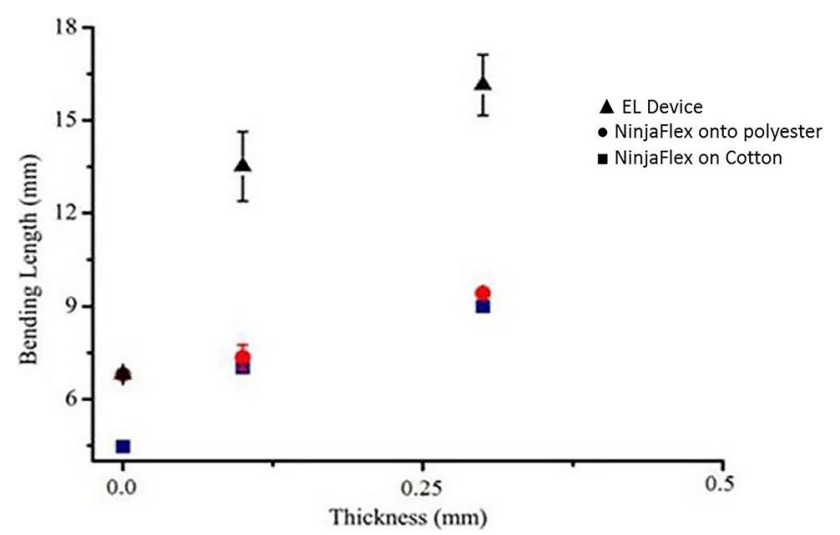

Fig. 5. Bending length measurement results.

samples with 0.1-mm and 0.3-mm thickness for both cotton and polyester fabrics. The relative bending lengths of the samples were increased by 0.577 and 0.0824 , respectively, for cotton and polyester fabrics with a $0.1-\mathrm{mm}$ thickness with respect to the fabric bending length. However, the relative bending lengths were increased by 1.0 and 0.387 for 0.3 mm-thick cotton and polyester fabric samples, respectively, which is relatively stiff. The relative bending lengths of the EL device were increased by 1.03 and 1.39 at $0.1-\mathrm{mm}$ and $0.3-\mathrm{mm}$ thickness of the NinjaFlex, respectively, when compared to bending lengths of the fabric. The results showed that the samples and the EL device maintain sufficient flexibility and that they can be easily integrated into wearable smart textiles, particularity with 0.1-mm-thick NinjaFlex, despite their adhesion strength.

The results showed that printing thickness and bending length were increased proportionally. However, this relationship is more evident when the measurement was taken on the side yielding the fabric (the upper side of the sample on the $3 \mathrm{D}$ printed portion); the 3D-printed samples maintained sufficient flexibility for integration into wearable textiles. The marginal increase in bending length is a highly desirable feature when considering incorporating 3D printing into the manufacture of wearable electronics, as it yields high clothing flexibility and freedom of movement.

In previous studies, various tests have been used to measure the adhesion of bonded objects, including peel strength testing, ${ }^{52-55}$ adhesion by tape test, ${ }^{56}$ and tear testing to evaluate the mechanical strength of the adhesive. ${ }^{57}$ In this study, the T-peel test was selected, and samples were prepared accordingly.

Three specimens at point ' $a$ ' for NinjaFlex and at point ' $b$ ' for the EL device were printed and tested for each sample (See Fig. 6). Specimens printed onto the polyester (point ' $a$ ') fabric were not fully delaminated. However, the NinjaFlex 3D-printed onto cotton (point ' $a$ ' and point ' $b$ ') was fully delaminated from the surface. Thus, the peel strength can be calculated from the average data using the equation below (Eq. 1). ${ }^{58}$ Peel strength (Newton/meter) has been defined as the average force $(F)$ per unit width $(W)$ required for separating the bonded objects. ${ }^{59}$

$$
\text { peel strength }(\mathrm{N} / \mathrm{m})=\frac{2 F}{W}
$$

Specimens printed onto polyester fabric yielded to the maximum load before fully delaminating, despite the samples not being completely peeled. NinjaFlex yields superior flexibility and longevity, with an elongation at a break of $660 \%$ according to the supplier information.

In the T-peel test, adhesion is defined as the energy per unit area required to separate NinjaFlex from cotton or polyester fabric. This implies that the highly elastic nature of the NinjaFlex could affect the results of adhesion. Thus, the maximum force required for separating the NinjaFlex from the fabric may be higher than the recorded value, as ascertained from energy balance considerations, the mechanical interlocking between the NinjaFlex and the fact that the fabric remains strong, as evidenced by the high peel strength. BendLay filament penetrated through the loop of the knitted copper fabric and made contact with phosphor paste that was coated onto the NinjaFlex (see Fig. 6). Statistically, the difference in the adhesion forces of NinjaFlex with both fabrics was insignificant. However, due to environmental resistance, polyester fabric was selected for the EL device fabrication. Therefore, the adhesion strength between the copper fabric and phosphor paste depended on the 3D-printed BendLay filament. Thus, as shown in Table I, the peeling strength recorded was sufficient to construct the EL device.

In addition to the mechanical adhesion, the resistance of the NinjaFlex against washing detergents is another important parameter for wearable devices. Performing washing durability tests determines whether washing detergents impact the 3Dprinted NinjaFlex and consequently affect adhesion. Three cycles of laundry washing tests were performed, and the results showed that no weight loss was recorded.

The washing performance characteristic of NinjaFlex printed onto textile fabric was a good indicator of adhesion durability on the textile surface, and it determines the integration of NinjaFlex with washable garments.

The schematic view of the device is shown in Fig. 7, where a phosphor blue paste layer can be seen sandwiched between 3D-printed NinjaFlex and copper fabric.

When an AC voltage of approximately $12 \mathrm{~V}$ was applied between the PEDOT:PSS-coated textile and translucent electrode (copper fabric), blue EL light 


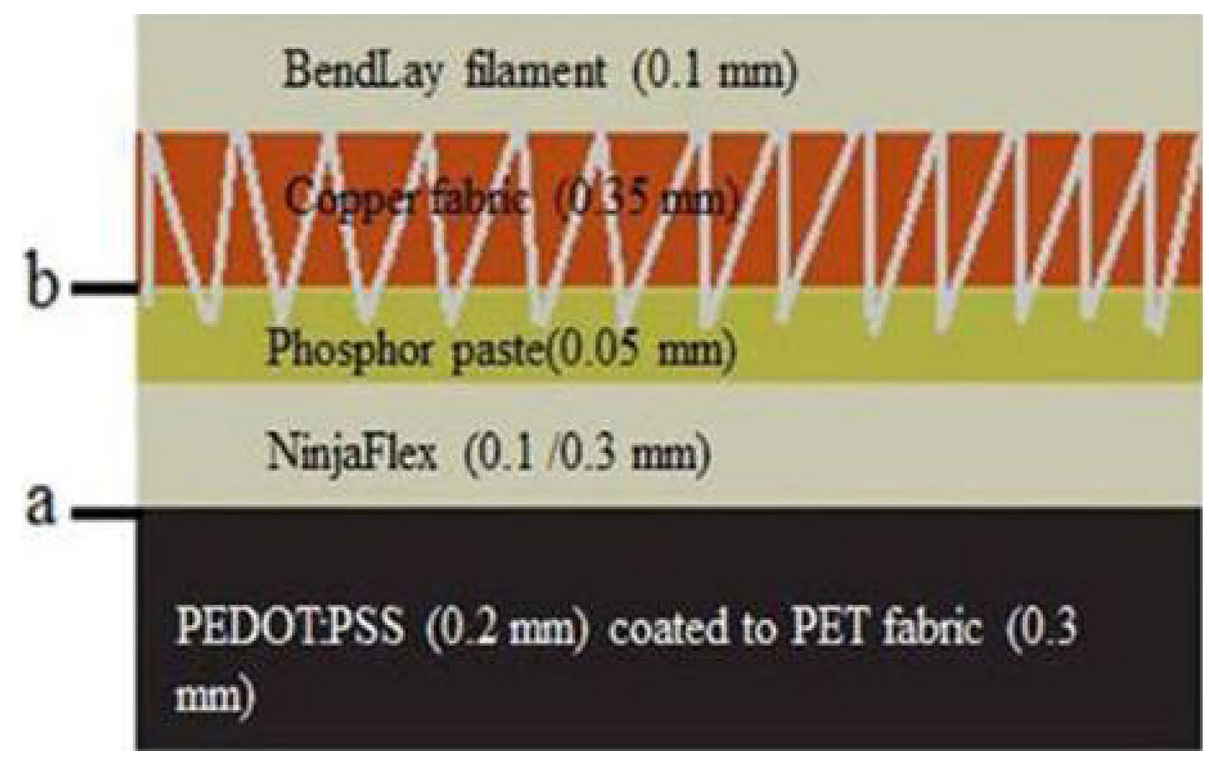

Fig. 6. Front views of the samples for adhesion testing of (a) NinjaFlex and (b) BendLay filament.

Table I. Maximum force and peel strength

\begin{tabular}{|c|c|c|c|c|c|}
\hline Sample & Thickness (mm) & Average force (N) & STD & Peel strength $(\mathrm{N} / \mathrm{m})$ & Remark \\
\hline Polyester & 0.3 & 52 & 4.4 & 4160 & $a^{\mathrm{a}}$ \\
\hline Cotton & 0.3 & 52 & 6.5 & 3840 & $a$ \\
\hline Copper & 0.1 & 39.4 & 14.5 & 3150 & $b^{\mathrm{b}}$ \\
\hline
\end{tabular}

${ }^{\mathrm{a} A s}$ indicated in Fig. $6 \mathrm{a} .{ }^{\mathrm{b}}$ As indicated in Fig. $6 \mathrm{~b}$.

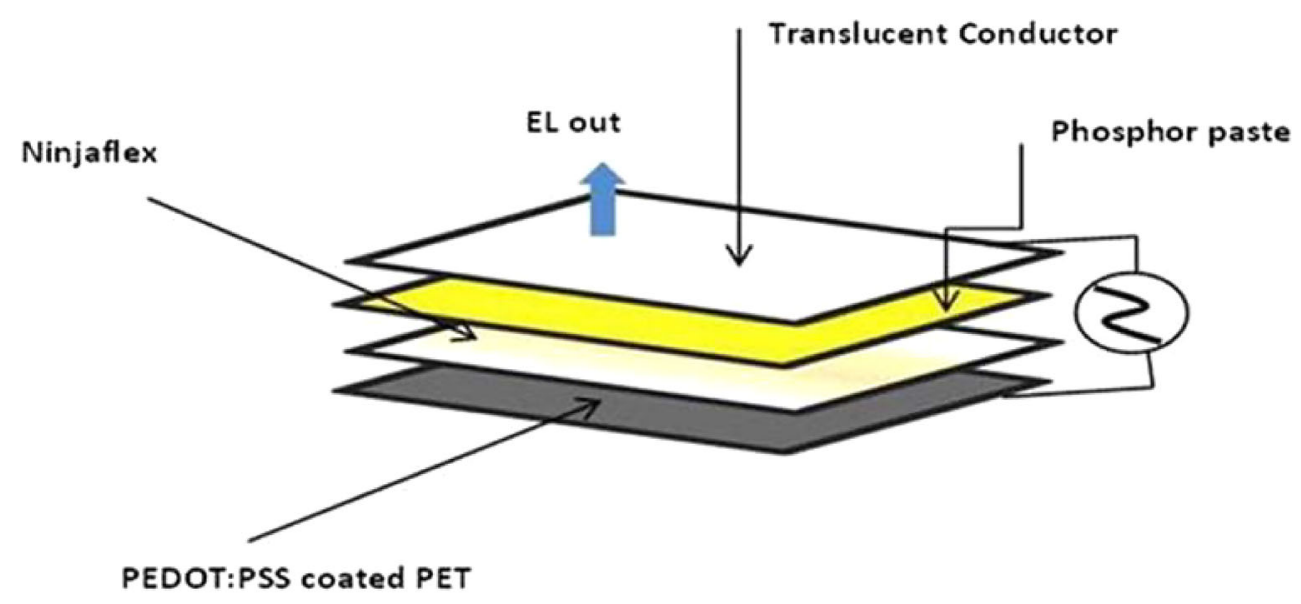

Fig. 7. Electroluminescence device fabrication method.

emission was observed despite EL having been observed when an AC voltage of approximately 50-200 V was applied across the anode and cathode, as claimed in Ref. 45. The materials used for insulation should have critical properties such as high dielectric constant and adhesion. NinjaFlex has excellent adhesion to the substrate with a dielectric permittivity around 3.5 at $100 \%$ infill percentage as suggested in Ref. 40. Dielectric constant is a numerical value used to define dielectric permittivity of an insulator material.

The EL light emission was investigated by connecting the anode and the cathode with an external power source of $12 \mathrm{~V}$ at $50-60 \mathrm{~Hz}$. Since the light 


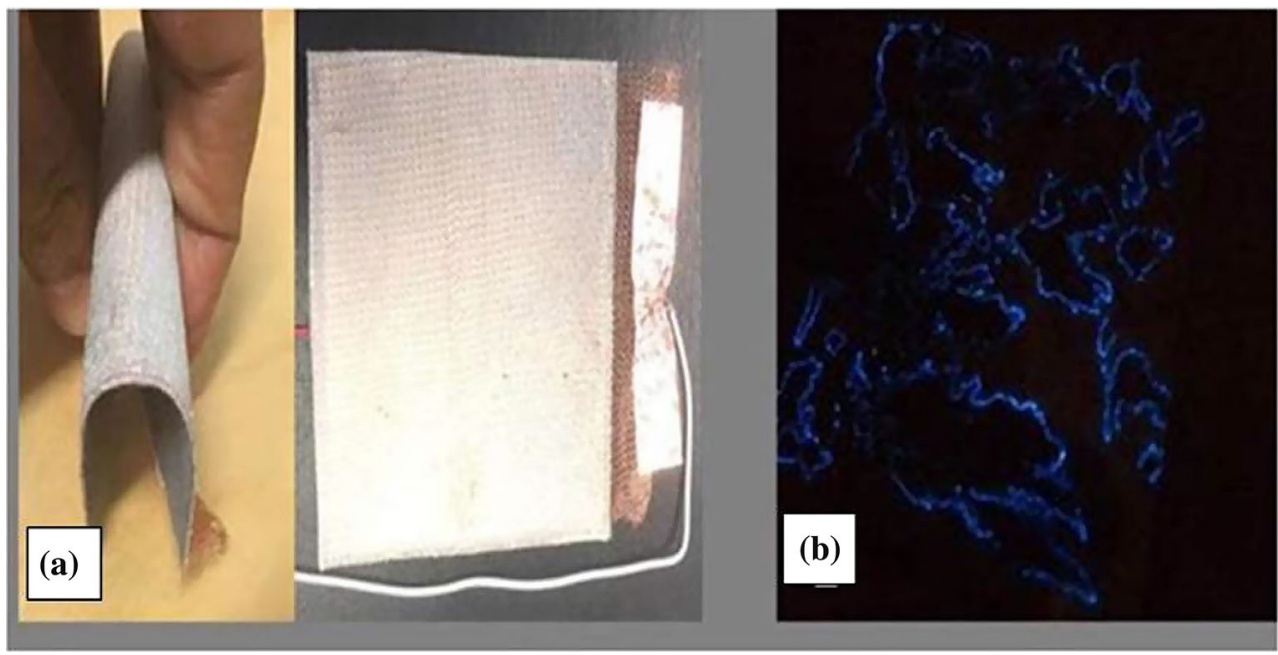

Fig. 8. Prototype of (a) EL device and (b) EL.

signal of the EL is so weak, the demonstration was performed in a dark environment to avoid inclusion of ambient light signals. The result is shown in Fig. 8b. These small blue light traces were detected when the applied electric field was greater than the threshold electric field, as claimed by Kido et al. ${ }^{45}$

Simple bending tests were performed to observe the flexibility of the device; results (see Fig. 8a) confirmed excellent performance absent of structural damage and cracking of the PEDOT:PSS. The structure was highly flexible and no cracks were observed on the phosphor paste, as the thickness of the paste was exceedingly thin and the materials were properly printed using methods of $3 \mathrm{D}$ printing and coating technology. The dielectric material, NinjaFlex, was unaffected by the bending action; this is attributed to the highly flexible and stretchable nature of the filament. The conductivity of the coated fabric was unchanged after several bending actions. This may be due to structural help from the 3D printing of NinjaFlex, copper fabric, and BendLay filament.

Although light emission was achieved, discontinuity of the emitted light proved to be one of the limitations of this prototype and can be a possible topic of future research. This may be due to the reduced contact area between the copper fabric and phosphor pastes, as higher force is required to achieve and maintain contact. The translucent filament, BendLay, was used to achieve full contact between the front electrode and emitter; however, the translucent filament 3D-printed on top the copper fabric was found to affect the conductivity of the copper fabric even with the increased contact area. Thus, further research is needed to enhance the contact area between the front electrode and a light emitter.

This work proposes a possible method of EL fabrication via incorporation of 3D printing and coating technologies. This method of EL device production may provide textile researchers with a foundation to extend current designs, which could better satisfy requirements of a variety of applications while also enabling customization of interactive designs and prints for smart and wearable textiles applications. Although the device exhibited discontinuous light emission, it is lightweight, robust with bending and folding, and highly luminescent in a dark environment.

\section{CONCLUSIONS}

This paper presented concepts of EL device fabrication using coating and 3D printing to fabricate rear electrodes with sufficient conductivity and dielectric materials, aiming to create a cost-effective suitable design for EL applications. Prototype samples made via coating and 3D printing were fabricated using a simple and cost-effective process for integration into wearable electronics. Given the stringent properties of rigid EL devices, an EL device with lightweight and flexible materials was fabricated. TGA of the dielectric material indicated that the material exhibits thermal stability up to $150^{\circ} \mathrm{C}$. The peel strengths of the 3D-printed NinjaFlex on the polyester and cotton fabrics were $4160 \mathrm{~N} / \mathrm{m}$ and $3840 \mathrm{~N} / \mathrm{m}$, respectively, which indicates that, with respect to fabric surfaces, NinjaFlex yields adhesive properties sufficient for use in EL applications. 3D-printed NinjaFlex provided excellent durability against repeated laundry washing. The relative increases in bending lengths of the EL device were 1.03 and 1.39 at $0.1-\mathrm{mm}$ and $0.3-\mathrm{mm}$ thicknesses of the NinjaFlex, respectively. Light emission was possible with an AC voltage as low as $12 \mathrm{~V}$ in a dark environment. The device could yield a wide variety of potential applications in entertainment and nightlife industries or advertising, as decorative luminescent clothing and other lightemitting devices. Furthermore, this NinjaFlex may enable additional smart textile applications in areas such as sensor and antenna fabrication due to its 
washability and strong adhesion to cotton and polyester fabrics.

Further studies may include efforts such as optimization of EL, V-I ohmic behavior of the device, EL lifetime stabilization against the environment, and EL characterization.

\section{ACKNOWLEDGEMENTS}

This research was supported by Erasmus Mundus, SMDTex Grant No. 2015-1594/001-001-EMJD. The authors would like to thank Desalegn Alemu and Molla Tadesse for SEM image measurement.

\section{CONFLICT OF INTEREST}

The authors declare no potential conflicts of interest with respect to this research or authorship of the article.

\section{OPEN ACCESS}

This article is distributed under the terms of the Creative Commons Attribution 4.0 International License (http://creativecommons.org/licenses/by/4.0/), which permits unrestricted use, distribution, and reproduction in any medium, provided you give appropriate credit to the original author(s) and the source, provide a link to the Creative Commons license, and indicate if changes were made.

\section{REFERENCES}

1. X. Jin and K. Gong, J. Ind. Text. 26, 34 (1996).

2. A.C. Sparavigna, L. Florio, J. Avloni, and A. Henn, Mater. Sci. Appl. 1, 252 (2010).

3. A. Bedeloglu, A. Demir, Y. Bozkurt, and N.S. Sariciftci, Text. Res. J. 80, 1065 (2010).

4. D.R. Cairns and G.P. Crawford, in IEEE Proceedings (2005), pp. 1451-1458.

5. W. Zeng, L. Shu, Q. Li, S. Chen, F. Wang, and X.M. Tao, Adv. Mater. 26, 5310 (2014).

6. Y. Ding, M.A. Invernale, and G.A. Sotzing, ACS Appl. Mater. Interfaces 2, 1588 (2010).

7. D.J. Lipomi, J.A. Lee, M. Vosgueritchian, B.C.K. Tee, J.A. Bolander, and Z.A. Bao, Chem. Mater. 24, 373 (2012).

8. C. Yeon, G. Kim, J. Lim, and S. Yun, RSC Adv. 7, 5888 (2017).

9. C.W. Tang and S.A. VanSlyke, Appl. Phys. Lett. 51, 913 (1987).

10. C. Adachi, K. Nagai, and N. Tamoto, Appl. Phys. Lett. 66, 2679 (1995).

11. M. De Vos, R. Torah, and J. Tudor, Smart Mater. Struct. 25, 045016 (2016).

12. T. Peng, Y. Yang, H. Bi, Y. Liu, Z. Hou, and Y. Wang, J. Mater. Chem. 21, 3551 (2011).

13. L.S. Sapochak, A. Padmaperuma, N. Washton, F. Endrino, G.T. Schmett, J. Marshall, D. Fogarty, P.E. Burrows, and S.R. Forrest, J. Am. Chem. Soc. 123, 6300 (2001).

14. G. Longo, A. Pertegás, L. Martínez-Sarti, M. Sessolo, and H.J. Bolink, J. Mater. Chem. 3, 11286 (2015).

15. L. Gil-Escrig, G. Longo, A. Pertegás, C. Roldán-Carmona, A. Soriano, M. Sessolo, and H.J. Bolink, Chem. Commun. 51,569 (2015).

16. L. Akcelrud, Prog. Polym. Sci. 28, 875 (2003).

17. I.F. Perepichka and D.F. Perepichka, Handbook of Thiophene-Based Materials: Applications in Organic Electronics and Photonics, 1st ed. (Chichester: John Wiley \& Sons, 2009), pp. 255-288.
18. M. De Vos, R. Torah, M. Glanc-Gostkiewicz, and J. Tudor, J. Disp. Technol. 12, 1757 (2016).

19. M.P. Aleksandrova, Microelectron. Int. 33, 47 (2016).

20. B. Hu, D. Li, O. Ala, P. Manandhar, Q. Fan, D. Kasilingam, and P.D. Calvert, Adv. Funct. Mater. 21, 305 (2011).

21. D. Zhu, X. Lu, and Q. Lu, Langmuir 30, 4671 (2014).

22. W. Hong, Y. Xu, G. Lu, C. Li, and G. Shi, Electrochem. Commun. 10, 1555 (2008).

23. Y.H. Kim, C. Sachse, M.L. Machala, C. May, L. MüllerMeskamp, and K. Leo, Adv. Funct. Mater. 21, 1076 (2011).

24. Y. Xia and J. Ouyang, J. Mater. Chem. 21, 4927 (2011).

25. A. Endo, M. Ogasawara, A. Takahashi, D. Yokoyama, Y. Kato, and C. Adachi, Adv. Mate. 21, 4802 (2009).

26. D.K. Fekety, D.E. Edewaard, A.A.S. Sewall, and R.A. Tyrrell, Hum. Factors 58, 976 (2016).

27. D. Bradley, Curr. Opin. Solid State Mater. Sci. 1, 789 (1996).

28. S.C. Yu, C.C. Kwok, W.K. Chan, and C.M. Che, Adv. Mater. 15, 1643 (2003).

29. J.A. Rogers, Z. Bao, and L. Dhar, Appl. Phys. Lett. 73, 294 (1998).

30. D.A. Skwarek, M. Sloma, D. Janczak, G. Wroblewski, A. Mlozniak, and M. Jakubowska, Circuit World 40, 13 (2014).

31. T. Ahn, S.Y. Song, and H.-K. Shim, Macromolecules 33, $6764(2000)$

32. I. Kazani, C. Hertleer, G. De Mey, A. Schwarz, G. Guxho, and L.V. Langenhove, Fibres Text. East. Eur. 20, 57 (2012).

33. W.C. Smith, Smart Textile Coatings and Laminates, 1st ed. (New York: Woodhead, 2010), pp. 155-184.

34. S.M. Bidoki, D. McGorman, D.M. Lewis, M. Clark, G. Horler, and R.E. Miles, AATCC Rev. 5, 11 (2005).

35. L. Hu, M. Pasta, F.L. Mantia, L. Cui, S. Jeong, H.D. Deshazer, J.W. Choi, S.M. Han, and Y. Cui, Nano Lett. 10, 708 (2010).

36. N.G. Tanikella, B.T. Wittbrodt, and J.M. Pearce, Addit. Manuf. 15, 40 (2017).

37. S. Moscato, R. Bahr, T. Le, M. Pasian, M. Bozzi, L. Perregrini, and M.M. Tentzeris, IEEE Antennas Wirel. Propag. Lett. 15, 1506 (2016).

38. T. Le, B. Song, Q. Liu, R.A. Bahr, S. Moscato, C.P. Wong, and M.M. Tentzeris, in 2015 IEEE 65th Electronic Components and Technology Conference (2015), pp. 981-986.

39. K. Nate and M.M. Tentzeris, in Electrical Performance of Electronic Packaging and Systems (2015), pp. 171-174.

40. E. Massoni, L. Silvestri, M. Bozzi, L. Perregrini, G. Alaimo, S. Marconi, and F. Auricchio, in Advanced Materials and Processes for RF and THz Applications IEEE MTT-S International Microwave Workshop Series (2016), pp. 1-4.

41. K. Bal and V.K. Kothari, Indian J. Fibre Text. Res. 34, 191 (2009).

42. M.C. Yuen and R.K. Kramer, in ASME 2016 11th International Manufacturing Science and Engineering Conference (2016), p. V002T01A014.

43. J. Sarik, A.I. Akinwande, and I. Kymissis, IEEE Trans. Educ. 54, 314 (2011).

44. J. Kido, M. Kimura, and K. Nagai, Science 267, 1332 (1995).

45. D.R. Vij, Handbook of Electroluminescent Materials (London: IOP Publishing, 2004), pp. 1-21.

46. C. Adachi, S. Tokito, T. Tsutsui, and S. Saito, Jpn. J. Appl. Phys. 27, L713 (1988)

47. C.W. Tang, J. Soc. Inf. Disp. 5, 11 (1997).

48. M.G. Tadesse, C. Loghin, Y. Chen, L. Wang, D. Catalin, and V. Nierstrasz, Smart Mater. Struct. 26, 065016 (2017).

49. M. Zahid, E.L. Papadopoulou, A. Athanassiou, and I.S. Bayer, Mater. Des. (2017). https://www.doi.org/10.1016/ j.matdes.2017.09.026.

50. J.D. Menczel and R.B. Prime, Thermal Analysis of Polymers: Fundamentals and Applications, 1st ed. (New York: Wiley, 2014), pp. 241-314.

51. X. Wang, X. Liu, and C. Hurren, Fabric Testing, ed. J. Hu (New York: Woodhead, 2008), p. 90.

52. N. De Geyter, R. Morent, F. Axisa, E. De Leersnyder, C. Leys, J. Vanfleteren, N. De Smet, M. Rymarczyk-Machal, 
and E. Schacht, in 3rd International Congress on Cold Atmospheric Pressure Plasmas: Sources and Applications (2007), pp. 17-20.

53. G.J. Jorgensen, K.M. Terwilliger, J.A. DelCueto, S.H. Glick, M.D. Kempe, J.W. Pankow, F.J. Pern, and T.J. McMahon, Sol. Energy Mater. Sol. Cells 90, 2739 (2006).

54. B.T. Poh and H.K. Kwo, J. Appl. Polym. Sci. 105, 680 (2007).

55. C. Spadaro, C. Dispenza, and C. Sunseri, Int. J. Adhes. Adhes. 28, 211 (2008)
56. ASTM D, 3359-02: Standard Test Methods for Measuring Adhesion by Tape Test (West Conshohocken, PA: ASTM International, 2002).

57. E. Hamm, P. Reis, M. LeBlanc, B. Roman, and E. Cerda, Nat. Mater. 7, 386 (2008).

58. M.J. Shenton, M.C. Lovell-Hoare, and G.C. Stevens, J. Phys. D Appl. Phys. 34, 2754 (2001).

59. ASTM D, 907-05: Standard Terminology of Adhesives (Annual Book of ASTM Standards, 2005). 